\title{
Preaccelerator concepts for an energy-recovering superconducting accelerator
}

\author{
Robert Heine $\odot^{*}$ \\ Johannes Gutenberg-Universität Mainz, Institut für Kernphysik, \\ Johann-Joachim-Becher-Weg 45, D-55128 Mainz, Germany
}

(Received 17 September 2020; accepted 14 December 2020; published 26 January 2021)

\begin{abstract}
In this paper we compare two concepts of a preaccelerator for the energy-recovery linac MESA: a hybrid injector that combines normal conducting and superconducting technology and a fully normal conducting injector. The particle source delivers polarized electrons at $100 \mathrm{keV}$. Thus the first accelerating section has to be of a graded- $\beta$ kind. The graded- $\beta$ section is designed with a final energy exceeding $1 \mathrm{MeV}$ so TESLA-type nine-cell resonators can be used as superconducting cavities. The final energy of the preaccelerator shall be $5 \mathrm{MeV}$ to avoid neutron production in the beam dump of the energy-recovery linac.
\end{abstract}

DOI: 10.1103/PhysRevAccelBeams.24.011602

\section{INTRODUCTION}

At Johannes Gutenberg-Universität Mainz the Institut für Kernphysik (KPH) is currently building an energy-recovery linac called MESA. MESA is the abbreviation for Mainz energy-recovering superconducting accelerator. It shall provide an electron beam for particle physics experiments at a duty factor of $100 \%$.

One of the two main experiments is $\mathrm{P} 2$ that measures the weak mixing angle at high precision via parity violation [1]. For this experiment MESA is running in external beam (EB) mode. This means the beam is dumped after the experiment in a beam stopper. P2 demands a beam of spin polarized electrons at a beam current of $I_{b}=150 \mu \mathrm{A}$, which translates to a bunch charge of $Q_{b}=0.12 \mathrm{pC}$, and with an energy of $T=$ $155 \mathrm{MeV}$ at a relative energy spread of the order of $10^{-4}$. The other experiment uses an internal gas jet target and is called MAGIX (MESA gas internal target experiment). MAGIX researches a variety of topics, e.g., proton radius and dark photons [2]. MESA is operated in energyrecovery (ER) mode for MAGIX to provide $I_{b}=1 \mathrm{~mA}$ $\left(Q_{b}=0.77 \mathrm{pC}\right)$ at $T=105 \mathrm{MeV}$. In an upgrade stage MESA is planned to provide $I_{b}=10 \mathrm{~mA}\left(Q_{b}=7.7 \mathrm{pC}\right)$ for MAGIX.

MESA (see Fig. 1) is a multiturn recirculating linac in race track geometry with vertically stacked arcs [3]. In each

\footnotetext{
"rheine@uni-mainz.de
}

Published by the American Physical Society under the terms of the Creative Commons Attribution 4.0 International license. Further distribution of this work must maintain attribution to the author(s) and the published article's title, journal citation, and DOI. straight section a cryostat equipped with two TESLAtype cavities [4] provides an energy gain of $25 \mathrm{MeV}$ in total. The cryostats are similar to those of ELBE at Helmholtz Zentrum Dresden Rossendorf [5] with some modifications [6] to adapt them for the use in MESA. In EB mode the beam passes each cavity 3 times, in ER mode the beam passes the cavities 4 times: twice accelerating and twice decelerating. The beam is then dumped at injection energy. MAGIX resides in an extension of the fourth MESA arc, for EB mode this part is bypassed.

The particle source of MESA is the dc photogun STEAM (small thermalized electron source at Mainz) that can produce polarized electrons at $100 \mathrm{keV}$ [7-9]. STEAM is driven by a radio frequency (rf) synchronized pulsed laser (for bunch length see Table I). Following STEAM the low energy beam transport line MELBA (MESA low energy beam apparatus) [10,11] leads the particles to the first rf cavity of the preaccelerator MAMBO (milliampere booster). MELBA is a rather long beam line of $\approx 9 \mathrm{~m}$, because it hosts a spin manipulation system consisting of two Wien filters [12] and a solenoid, a chopper system consisting of two circular deflecting cavities and a collimator with movable jaws and a buncher system that has a fundamental and a second harmonic cavity.

The second resonator of the chopper removes the energy modulation introduced by the first one [13]. Only a small residue of circa $10 \%$ of this modulation is left. This is a higher order effect of the beam diameter inside the second cavity. The harmonic buncher linearizes the phase space distribution. Therefore it acts as a debuncher that is separated from the fundamental buncher by a drift space of an odd integer multiple of the $\mathrm{rf}$ 


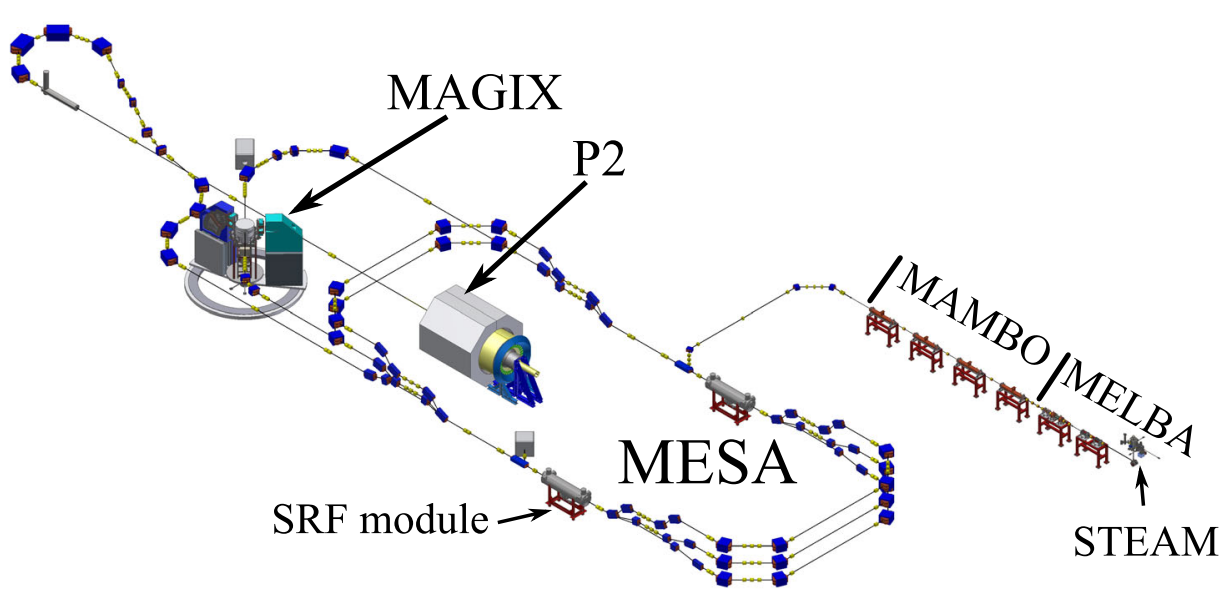

FIG. 1. Overview of the planned MESA facility.

wavelength [14]. The effect of the harmonic buncher can be seen quite nicely in Fig. 2(a). The use of the harmonic buncher allows having the chopper accept a rather large phase spread. So most of the electrons from the source are accelerated and only halo is rejected. This is important since the GaAs photocathodes used have a limited amount of charge of $Q_{\max }=700 \mathrm{C}$ [15] that can be extracted before they need to be refreshed.

MELBA uses solenoids and quadrupole triplets for transverse focusing. Quadrupoles are necessary to compensate fringe field effects of the Wien filters. The solenoids are split in two parts, whose field orientation is alternating between parallel and antiparallel to the beam direction in order to cancel out spin precession.

\section{A PRIORI CONSIDERATIONS}

After considering different cryostat options also at different rf frequencies for MESA [16] the ELBE module was chosen for the main accelerator. So the frequency was fixed to $f_{\mathrm{rf}}=1.3 \mathrm{GHz}$. The preaccelerator could operate at the same frequency or a harmonic. At a harmonic frequency the duty cycle needs to be reduced accordingly, which is easily achievable. But the shorter wavelength at a harmonic frequency implies a higher peak current at a given bunch charge and therefore a higher space charge field. To avoid this, the frequency of MAMBO will also be $1.3 \mathrm{GHz}$.

It was chosen to operate the particle source at $100 \mathrm{keV}$ for several reasons. Most prominent the negative electron affinity photocathodes necessary to generate spin-polarized electrons are rather delicate. Experience with the MAMI particle source $[17,18]$ during the past 3 decades showed them to be very reliable at this energy. Since MESA shall be a production machine providing several thousand hours of operation for data taking per year, reliability is most important. Further the setups for spin manipulation and bunch preparation are more compact at lower $\beta$.
The beam stop of the main accelerator shall be placed inside the hall with modest shielding. Electrons exceeding energy of 3 to $10 \mathrm{MeV}$ can produce neutrons depending on the material of the beam stop [19]. To avoid neutron production at the beam dump the injection energy to MESA is going to be $5 \mathrm{MeV}$.

The energy of $100 \mathrm{keV}$ provided by the particle source translates to $\beta=0.548$. This low energy beam cannot be injected into high $\beta$ cavities, because a strong phase slip will occur. This is caused by a large difference in the velocities of the particles and the accelerating wave. Therefore it is necessary to use a graded- $\beta$ type structure as a first accelerating stage. There the length of each accelerating cell and thus the phase velocity of the $\mathrm{rf}$ wave is matched to the particle velocity. To our knowledge, there are no superconducting $1.3 \mathrm{GHz}$ booster cavities for electron acceleration in continuous wave $(\mathrm{cw})$ at such low $\beta$ available at the moment. KPH does not have the expertise to develop such resonators, but a long tradition in normal conducting $\mathrm{cw}$ standing wave acceleration at the Mainz microtron MAMI [20]. So it seems to be sensible to design at least the first accelerating stage with normal conducting technology. This stage shall provide at least a beam energy of $1 \mathrm{MeV}$. This translates to $\beta \approx 0.94$ which can be captured in a $\beta=1$ rf section sufficiently well. Now there are two options that shall be investigated in the following sections: a fully normal conducting linac and a hybrid linac that starts with a normal conducting graded- $\beta$ section and then uses, e.g., one or two TESLA-type nine-cell cavities to reach the final energy. Earlier design stages of MAMBO had been reported in [21-23].

All beam dynamics simulations have been carried out with PARMELA [24] using 3D space charge. The normal conducting structure has been modeled with SUPERFISH [25]. The cells are represented in PARMELA by their Fourier coefficients. A script to find optimum cell length for a given energy gain and phase slippage per cell was implemented 


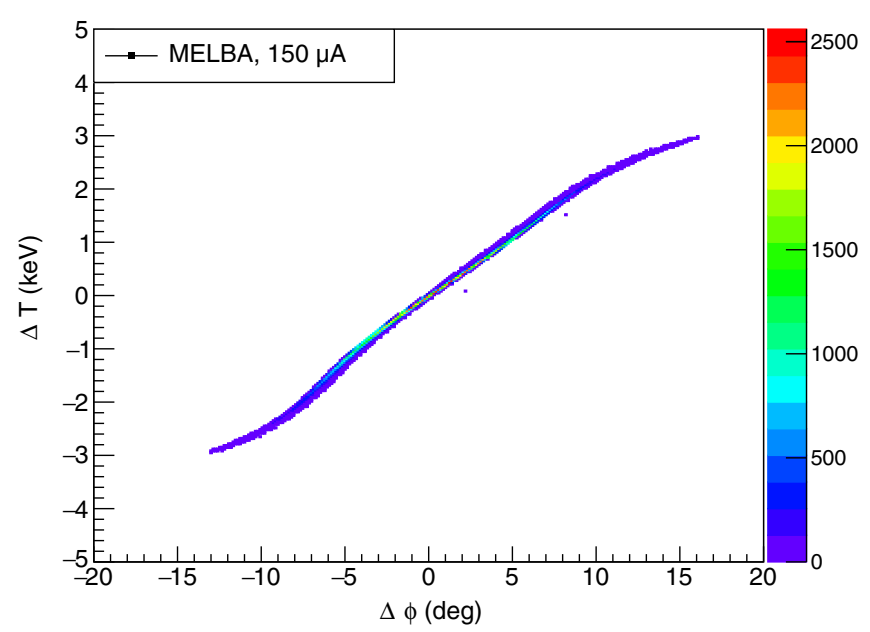

(a)

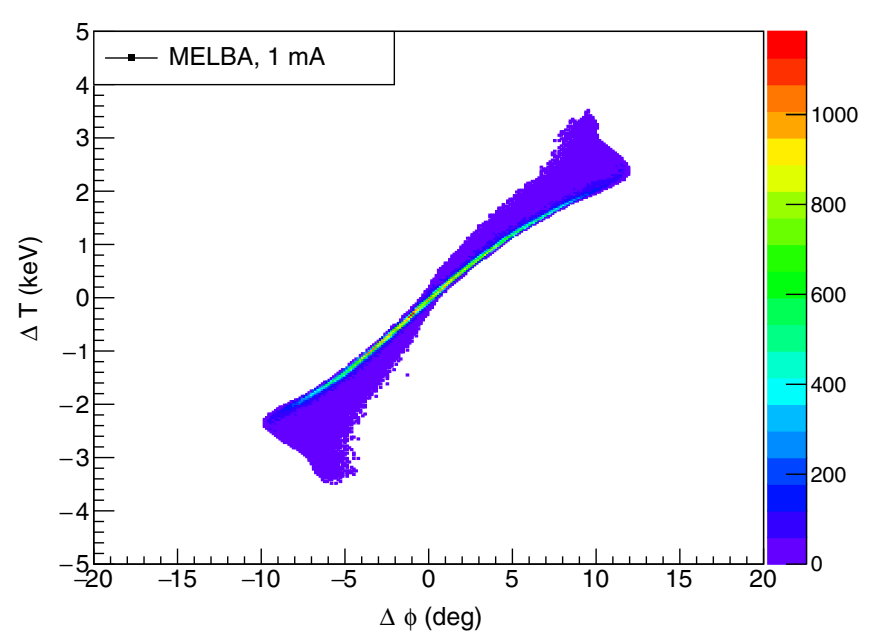

(b)

FIG. 2. Particle distributions in longitudinal phase space of different beam currents (Fig. 2a: $I_{b}=150 \mu \mathrm{A}$ and Fig. 2b: $I_{b}=1 \mathrm{~mA}$ ) at the end of MELBA that are injected into the first MAMBO rf section.

with the ROOT package [26]. This script interpolates the Fourier coefficients of the cell from a look-up table. The script is a reproduction of a function called "design" that had been part of earlier PARMELA versions. Both buncher cavities and the superconducting rf (SRF) cavities are represented by SUPERFISH field maps. Further the built-in chopper element is used and the acceptable phase spread is set to $\Delta \phi_{c}= \pm 80^{\circ}$.

The 6D input particle distributions to be used with PARMELA in the following were extracted from CST PARTICLE STUDIO simulations during the design of STEAM [9]. The longitudinal phase space projection of the particles at the end of MELBA as they are going to be injected into the first MAMBO section are shown in Fig. 2. The data presented in this publication has been extracted with the PARGRAF tool provided with PARMELA. The corresponding data is listed in Table I. Two beam
TABLE I. Bunch data of the $100 \mathrm{keV}$ beam as extracted from STEAM at $I_{b}=150 \mu \mathrm{A}$ and $I_{b}=1 \mathrm{~mA}$ and at the end of MELBA. The phase space distribution of the beam injected into MAMBO is shown in Fig. 2. The acceptable phase spread of the chopper was set to $\Delta \phi_{c}= \pm 80^{\circ}$. Additionally the transverse rmsemittance values are listed. Further the same data for the bunch exiting section MAMBO 1 is given. The longitudinal distributions are depicted in Fig. 3. At $I_{b}=1 \mathrm{~mA}$ solenoids over MAMBO 1 are engaged.

\begin{tabular}{|c|c|c|c|c|c|c|}
\hline \multirow[b]{2}{*}{$I_{b} \mathrm{~mA}$} & \multicolumn{2}{|c|}{ STEAM } & \multicolumn{2}{|c|}{ MELBA } & \multicolumn{2}{|c|}{ MAMBO 1} \\
\hline & 0.15 & 1 & 0.15 & 1 & 0.15 & 1 \\
\hline$\langle T\rangle \mathrm{MeV}$ & 0.1 & 0.1 & 0.1 & 0.1 & 1.279 & 1.279 \\
\hline$\Delta T_{\mathrm{rms}} \mathrm{keV}$ & 0.007 & 0.03 & 1.02 & 1.36 & 3.38 & 3.8 \\
\hline$\Delta T_{\mathrm{rms}} /\langle T\rangle 10^{-4}$ & 0.7 & 3.1 & 102 & 135.4 & 26.5 & 29.3 \\
\hline$\Delta \phi_{\mathrm{rms}}$ & 23.1 & 23.2 & 4.42 & 5.2 & 1.28 & 1.47 \\
\hline$\epsilon_{\mathrm{rms}}{ }^{\circ} \mathrm{keV}$ & 0.08 & 0.35 & 0.29 & 1.11 & 0.88 & 1.73 \\
\hline$\epsilon_{99 \%}{ }^{\circ} \mathrm{keV}$ & 1.41 & 6.39 & 4.39 & 18.4 & 14.83 & 25 \\
\hline$\epsilon_{100 \%}{ }^{\circ} \mathrm{keV}$ & 2.75 & 12.5 & 40.5 & 102.4 & 194.7 & 60.3 \\
\hline$\epsilon_{x, \mathrm{rms}} \mathrm{mm} \mathrm{mrad}$ & 0.113 & 0.12 & 0.16 & 0.66 & 0.18 & 1.3 \\
\hline$\epsilon_{y, \mathrm{rms}} \mathrm{mm} \mathrm{mrad}$ & 0.113 & 0.12 & 0.13 & 0.34 & 0.14 & 1.25 \\
\hline
\end{tabular}

currents were researched exemplarily: $I_{b}=150 \mu \mathrm{A}$ and $I_{b}=1 \mathrm{~mA}$.

\section{NORMAL CONDUCTING PREACCELERATOR}

The normal conducting MAMBO rf structure is a biperiodic standing wave structure [27,28] with magnetic coupling operated in $\pi / 2$ mode. This design makes use of the fact that in this particular mode even numbered cells have no field and therefore can be shortened to increase accelerating efficiency, i.e., shunt impedance. The cells are named after their function: those with field are called accelerating cells (AC) and the shortened ones coupling cells (CC).

Biperiodic structures at 2.45 and $4.9 \mathrm{GHz}$ are in use at MAMI for a long time. The MAMI injector linac ILAC has a cell design optimized for $\beta$ grading [29]. To be used at MAMBO this design was scaled to $1.3 \mathrm{GHz}$ and modified to allow for higher cell coupling and a larger beam pipe diameter. The later was found necessary, because the beam current of MAMBO shall be a factor 10 to 100 larger than that of ILAC.

The normal conducting MAMBO consists of four accelerating sections. The first one is a graded- $\beta$ section (MAMBO 1) where the length of the cell with the index $g$ is $l_{g}$. The following ones are constant- $\beta$ sections (MAMBO 2-4). Due to shorter ACs the shunt impedance of MAMBO 1 is decreased considerably to $R_{s}=46.7 \mathrm{M} \Omega$ while the constant $\beta$ sections have $R_{s} / L=44.9 \mathrm{M} \Omega / \mathrm{m}$ which is $R_{s} \approx 100 \mathrm{M} \Omega$, each. In this paper the linac definition of shunt impedance is used. The rf sections are separated by short drift spaces that incorporate transverse focusing elements. Additionally six solenoids 
TABLE II. Length $l_{g}$ of the cells of the graded- $\beta$ rf section MAMBO 1. The lengths are measured from the center of the two neighboring coupling cells. $g$ is the index of the cell. There are 19 $\mathrm{AC}$ and thus $18 \mathrm{CC}$. $R_{s}=46.7 \mathrm{M} \Omega$, which corresponds to $P_{c}=41 \mathrm{~kW}$.

\begin{tabular}{rrrrrrrr}
\hline \hline \multicolumn{2}{l}{$\beta=0.579 \ldots 0.958$} \\
\hline $\mathrm{g}$ & $l_{g} / \mathrm{mm}$ & $\mathrm{g}$ & $l_{g} / \mathrm{mm}$ & $\mathrm{g}$ & $l_{g} / \mathrm{mm}$ & $\mathrm{g}$ & $l / \mathrm{mm}$ \\
\hline 1 & 66.8 & 6 & 93.6 & 11 & 104.2 & 16 & 108.8 \\
2 & 74.2 & 7 & 96.6 & 12 & 105.4 & 17 & 109.4 \\
3 & 80.4 & 8 & 99.0 & 13 & 106.4 & 18 & 110.0 \\
4 & 85.6 & 9 & 101.0 & 14 & 107.4 & 19 & 110.4 \\
5 & 90.0 & 10 & 102.8 & 15 & 108.2 & & \\
\hline \hline
\end{tabular}

producing $B_{\text {sol }}=10 \mathrm{mT}$ each are placed over MAMBO 1 . Using those allows weaker focusing magnets inside the drift spaces for currents around $I_{b}=1 \mathrm{~mA}$. The field direction of the MAMBO 1 solenoids is also alternating to

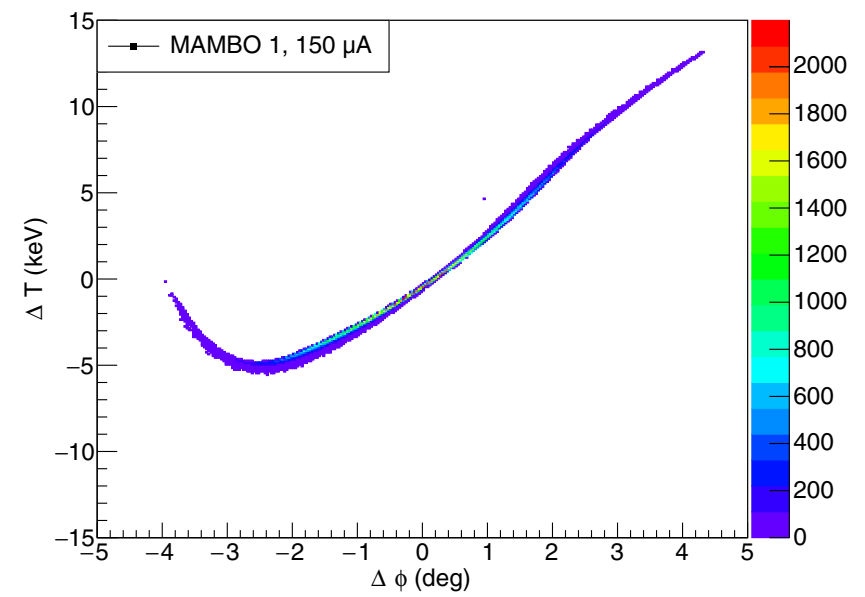

(a)

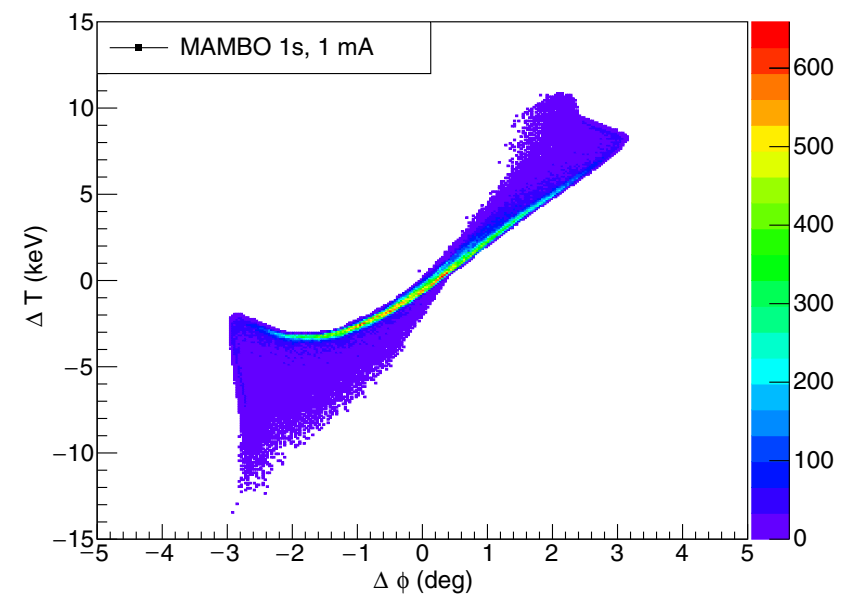

(b)

FIG. 3. Particle distributions in longitudinal phase space of different beam currents at the end of the first MAMBO RFsection. At $I_{b}=1 \mathrm{~mA}$ the solenoids over MAMBO 1 were used at $B_{\text {sol }}=10 \mathrm{mT}$ (indicated by 's' in the legend of Fig. 3b).

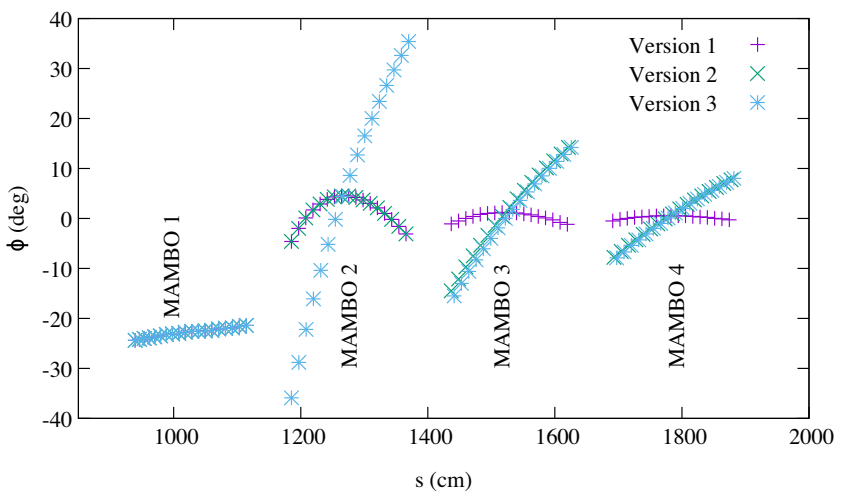

FIG. 4. Beam phases of the three versions of the normal conducting MAMBO.

compensate spin precession. Beam waists are avoided, because they increase beam divergence and space charge density.

MAMBO 1 is designed to deliver a final energy of $1.25 \mathrm{MeV}$ at a phase slippage per cell of $\Delta \phi=0.1^{\circ}$. The injection phase shall be $\phi=-25^{\circ}$ with respect to the maximum of the sine. This represents the phase convention at linacs. The Ohmic losses in MAMBO 1 are $P_{c}=41 \mathrm{~kW}$. The bunch is focused by the buncher system into the first rf section that follows after a drift of $1.5 \mathrm{~m}$. There the focal point lies approximately in the fifth cell. The bunch executes half a period of synchrotron motion in MAMBO 1 and leaves the section longitudinally converging. The focal point would be near the exit of MAMBO 3, if MAMBO 2 and 3 would not alter the focusing. The lengths of the cells $l_{g}$ of MAMBO 1 are listed in Table II. MAMBO 1 is kept the same for all variations presented here. The bunch data of the beam exiting MAMBO 1 is given in Table I. The longitudinal phase space diagrams are shown in Fig. 3.

The sections MAMBO 2-4 are designed to provide an energy gain of $1.25 \mathrm{MeV}$ each and be of less than $2 \mathrm{~m}$ length for practical purposes. The effective accelerating gradient at the $\mathrm{AC}$ is $E_{\mathrm{acc}} \approx 0.66 \mathrm{MV} / \mathrm{m}$. The power dissipated in each rf section is $P_{c} \approx 19 \mathrm{~kW}$. Now there are three variants to be investigated.

TABLE III. Length $l_{j}$ of the cells of the rf sections MAMBO 24 for different variations of the normal conducting concept. The lengths are measured from the center of the two neighboring coupling cells. The index $j$ indicates the section number. Each tank consists of $17 \mathrm{AC}$ and $16 \mathrm{CC}$. The shunt impedance is $R_{s} / L=44.9 \mathrm{M} \Omega / \mathrm{m}$, so $P_{c} \approx 19 \mathrm{~kW}$ for each one.

\begin{tabular}{|c|c|c|c|c|c|c|}
\hline & \multicolumn{2}{|c|}{ Version 1} & \multicolumn{2}{|c|}{ Version 2} & \multicolumn{2}{|c|}{ Version 3} \\
\hline & $\beta_{j}$ & $l_{j} / \mathrm{mm}$ & $\beta_{j}$ & $l_{j} / \mathrm{mm}$ & $\beta_{j}$ & $l_{j} / \mathrm{mm}$ \\
\hline & 0.977 & & 0.977 & & 1.0 & 115.3 \\
\hline MAI & 0.990 & 114. & 1.0 & 115.3 & 1. & 115.3 \\
\hline MAMBO 4 & 0.995 & 114.69 & 1.0 & 115.3 & 1.0 & 115.3 \\
\hline
\end{tabular}




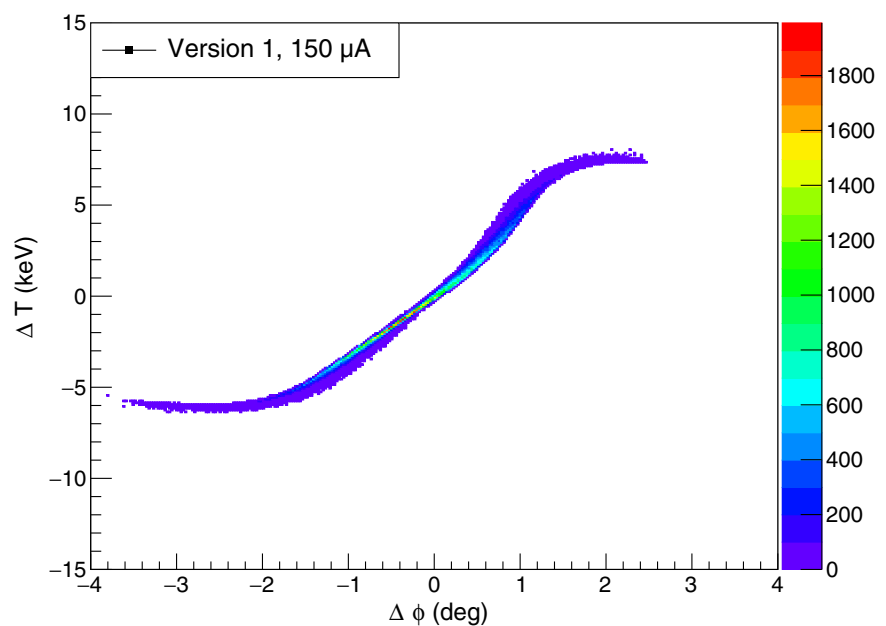

(a)

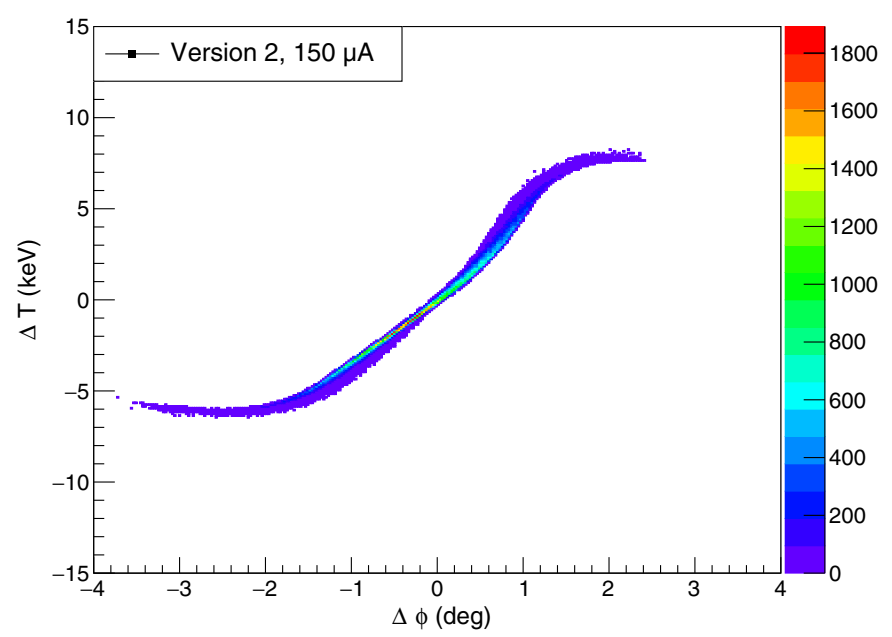

(c)

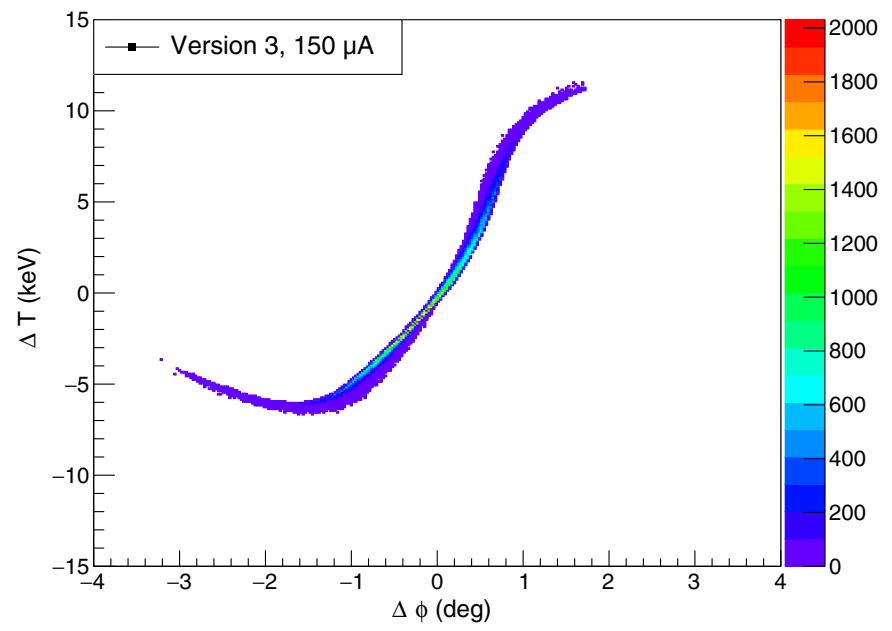

(e)

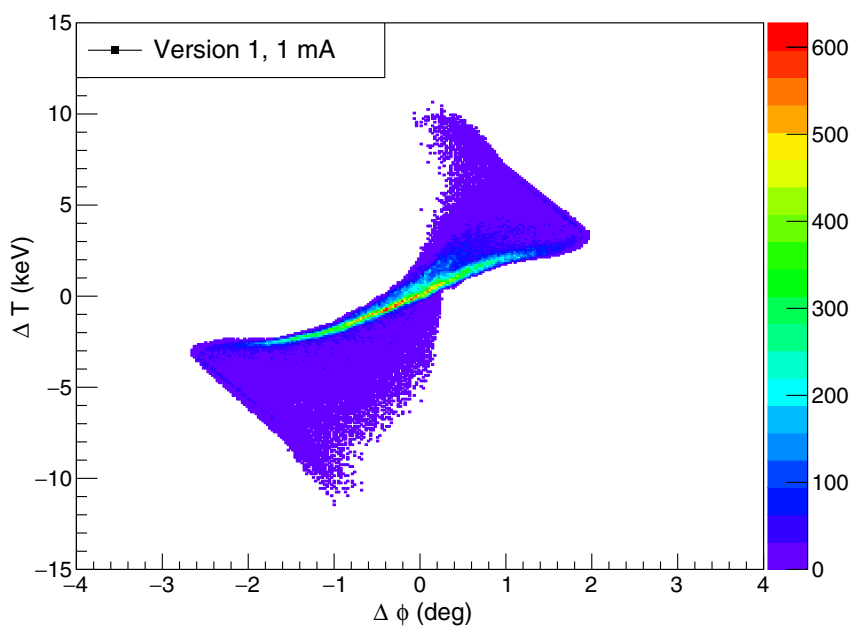

(b)

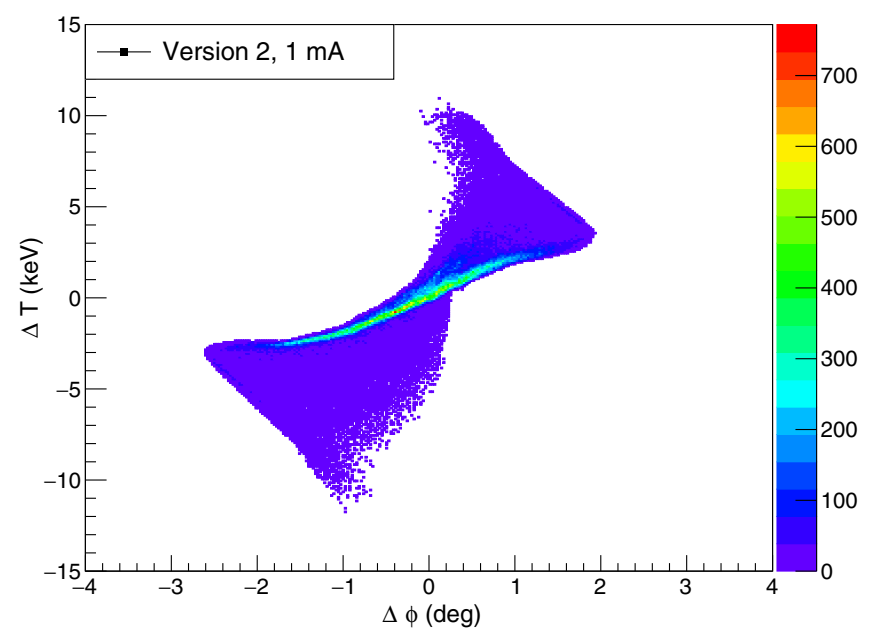

(d)

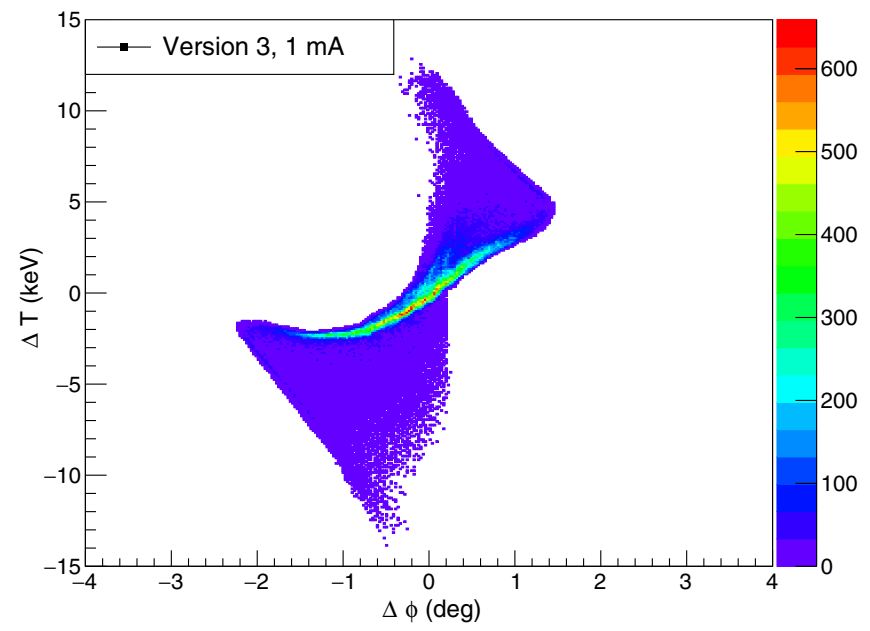

(f)

FIG. 5. Phase space distributions of the particles at the end of a normal conducting MAMBO at $I_{b}=150 \mu \mathrm{A}$ (Figs. 5a, 5c and 5e) and $I_{b}=1 \mathrm{~mA}$ (Figs. 5b, 5d and 5f). The figures of merit (bunch length, energy spread and emittances) of each version are listed in Table IV. 
(i) In the first version the lengths of the cells are $l_{j}=\beta_{j} \lambda_{\mathrm{rf}} / 2$. Now $\beta_{j}$ of each rf section $(j=2,3,4)$ is set to be in the middle between injection $\beta_{\text {in }}$ and extraction $\beta_{\text {out }}$ of that particular accelerating stage. The phase slippage is positive if the particle velocity is less and negative if it is more than $\beta_{j}$. The injection phases shall be negative, i.e., focusing. They are selected such that the absolute value of minimum and maximum phase are about the same (see Fig. 4). This also results in $\phi_{\text {in }} \approx \phi_{\text {out }}$ which is a kind of a longitudinal triplet focusing. This strategy has been adopted from the concept of ILAC.

(ii) In version 2 there is $\beta_{3}=1$ and $\beta_{4}=1$, too. From the point of easy fabrication of the resonators this seems to be sensible since in version 1 we have $\beta_{3} \approx 1$ and also $\beta_{4} \approx 1$. The slope of the phase slippage is always positive for $\beta=1$. The injection phase is chosen to be focusing. A good starting point is $\left|\phi_{\text {in }}\right| \approx\left|\phi_{\text {out }}\right|$. The amount of net focusing can be adjusted by shifting input phases. $\phi_{\text {in }}$ of MAMBO 2 is kept as in version 1 .

(iii) For completeness a third version is investigated where also $\beta_{2}=1$.

The corresponding $\beta$ and lengths of the cells are listed in Table III. The beam phases are plotted in Fig. 4. As one can see there, phases of identical rf sections are kept the same. This has been done for a better comparison of the effects caused by the changes introduced. Also the phases of MELBA have not been touched. The amplitudes of the buncher cavities at $I_{b}=1 \mathrm{~mA}$ have been raised by $20 \%$ to allow for a comparable bunch length at the entrance of MAMBO 1 (see Table I). Also some transverse focusing elements directly in front of MAMBO 1 have been altered to optimize beam transfer at $1 \mathrm{~mA}$.

The final phase space distributions as found $0.1 \mathrm{~m}$ behind the last rf section are shown in Fig. 5. The bunch data obtained from those distributions are listed in Table IV.

Each of the three versions researched can lead to a longitudinally converging particle distribution. So a short bunch at the first MESA cryostat can be obtained. The

TABLE IV. Comparison of longitudinal bunch data of the researched normal conducting MAMBO versions as found $0.1 \mathrm{~m}$ behind the end of the last $\mathrm{rf}$ section. For a complete picture the transverse rms emittances are also given.

\begin{tabular}{lcccccc}
\hline \hline & \multicolumn{2}{c}{ Version 1 } & \multicolumn{2}{c}{ Version 2 } & \multicolumn{2}{c}{ Version 3 } \\
\hline$I_{b} \mathrm{~mA}$ & 0.15 & 1 & 0.15 & 1 & 0.15 & 1 \\
$\langle T\rangle \mathrm{MeV}$ & 5.05 & 5.05 & 5.04 & 5.04 & 4.98 & 4.98 \\
$\Delta T_{\mathrm{rms}} \mathrm{keV}$ & 2.94 & 2.6 & 3.01 & 2.7 & 3.6 & 3 \\
$\Delta T_{\mathrm{rms}} /\langle T\rangle{ }^{\circ} 10^{-4}$ & 5.8 & 5.2 & 6 & 5.4 & 7.2 & 6.1 \\
$\Delta \phi_{\mathrm{rms}}{ }^{\circ}$ & 0.79 & 0.97 & 0.77 & 0.95 & 0.58 & 0.74 \\
$\epsilon_{\mathrm{rms}}{ }^{\circ} \mathrm{keV}$ & 0.4 & 1.26 & 0.41 & 1.26 & 0.58 & 1.34 \\
$\epsilon_{99 \%}{ }^{\circ} \mathrm{keV}$ & 5.63 & 20.25 & 5.74 & 20.3 & 8.53 & 19.93 \\
$\epsilon_{100 \%}{ }^{\circ} \mathrm{keV}$ & 112.7 & 74.4 & 115.9 & 74.6 & 146 & 68.7 \\
$\epsilon_{x, \mathrm{rms}} \mathrm{mm} \mathrm{mrad}$ & 0.22 & 1.32 & 0.22 & 1.32 & 0.21 & 1.32 \\
$\epsilon_{y, \mathrm{rms}} \mathrm{mm} \mathrm{mrad}$ & 0.15 & 1.21 & 0.15 & 1.21 & 0.14 & 1.21 \\
\hline \hline
\end{tabular}

actual distribution there is of course depending on the properties of the transfer channel to MESA and the actual phases chosen for the MAMBO rf sections. In a former concept [21] the linac was optimized for minimum energy spread. But research of injection into MESA showed that a short bunch is more desirable to achieve a good energy spread $\Delta T$ at the experiment than a low initial $\Delta T$ [30]. So the design goals were changed accordingly. For special purposes minimum $\Delta T$ can still be achieved by changing the phases of, e.g., MAMBO 4.

Looking at the phase space diagrams in Fig. 5, one can see that for $150 \mu \mathrm{A}$ the cores of the distribution of version 1 and 2 are still quite straight, while it is slightly bent upwards in version 3. All distributions show some s-formed tails that are a result of the unavoidable nonlinearity of $\mathrm{rf}$ voltage. At $I_{b}=1 \mathrm{~mA}$ the core of the distribution is wider and more distorted due to space charge. Also the tails have become larger and more spread in both directions, $\Delta T$ and $\Delta \phi$. The phase space distributions of versions 1 and 2 are nearly the same. From the diagrams Figs. 5(a) and 5(c) at $I_{b}=150 \mu \mathrm{A}$ and also from Figs. 5(b) and 5(d) at $I_{b}=1 \mathrm{~mA}$ they cannot be distinguished. Differences are mostly found at low current. Comparing the numbers in Table IV, especially the emittances, one could say that in version 2 they are slightly worse than in version 1 by approximately $2 \%$, while in version 3 there are up to $45 \%$ larger emittances than in version 1 . Remarkably version 3 delivers about $25 \%$ shorter bunches than version 1 . This can be understood from the strongly negative phases in the second rf tank. Also version 2 produces slightly shorter bunches than version $1(\approx 3 \%)$.

The transverse rms emittances of all versions do not differ considerably. Comparing data from Tables I and IV one can also see that most of the emittance growth happens during transport of higher beam current at low energy.

The simulations also show that versions 2 and 3 are reaching a reduced final energy without adapting the field amplitudes. This is due to the fact that in the $\beta=1$ sections acceleration deviates more from on-crest acceleration than in the corresponding cavities of version 1 .

Concluding the research of a normal conducting MAMBO the preaccelerator version 2 seems to be a good compromise of simplified fabrication and good bunch data.

\section{HYBRID PREACCELERATOR}

The hybrid MAMBO would be identical to the normal conducting concept up until the end of the drift space following the graded $-\beta$ section MAMBO 1 . Then a cryostat with SRF cavities can be used to reach the final energy. There are two advantages one can see from the beginning: much less rf power is needed and the preaccelerator will be much shorter.

Two versions are investigated: (i) Hybrid 1 utilizes a cryostat with one TESLA-type nine-cell cavity (one-seater), e.g., the TRIUMF ARIEL e-Linac injector cryomodule 


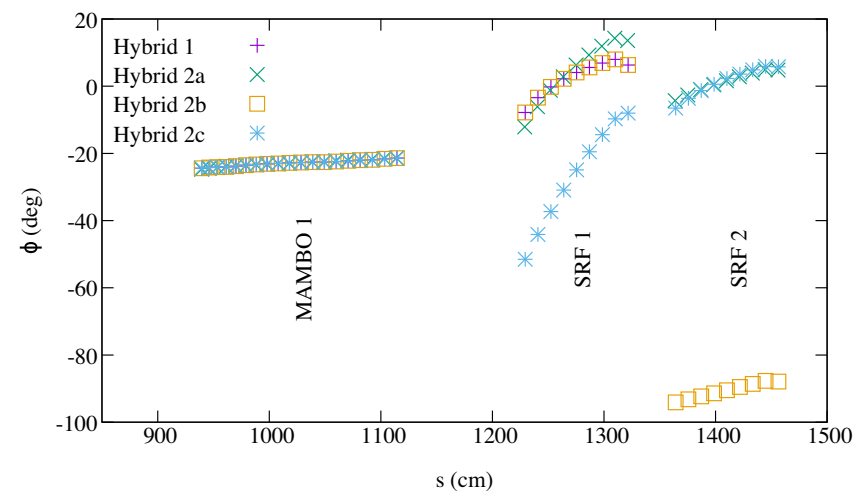

FIG. 6. Beam phases of the two versions of the hybrid MAMBO. For the two-seater cryomodule several modes of operation are shown.

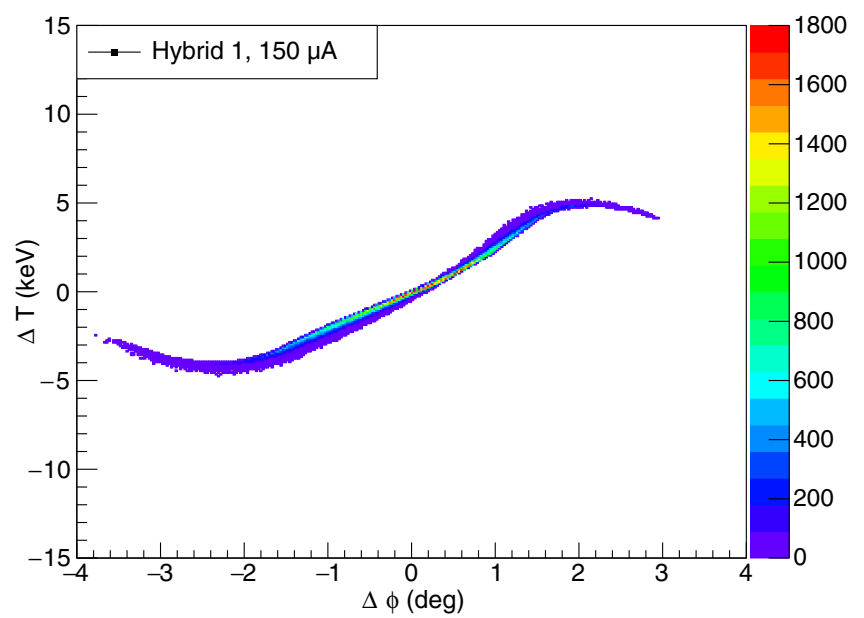

(a)

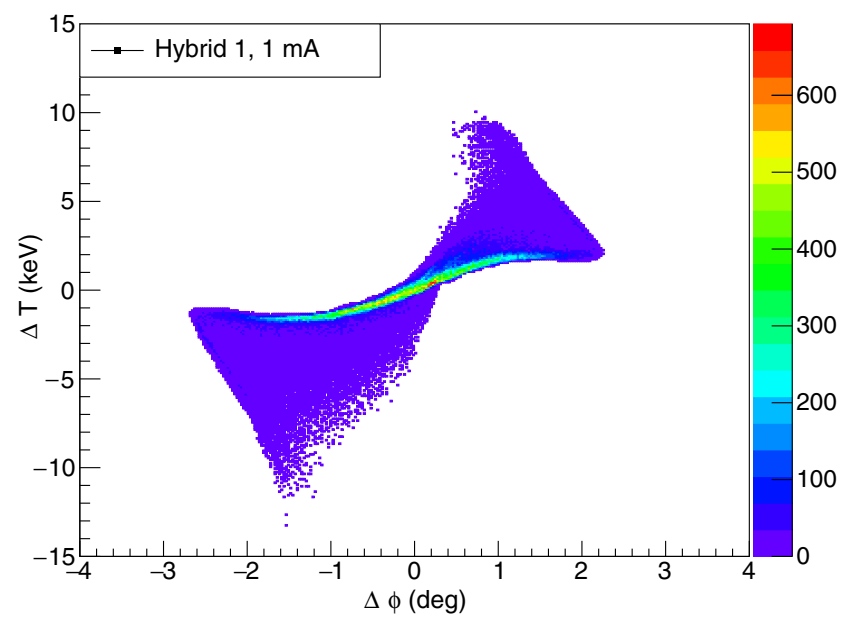

(b)

FIG. 7. Phase space distributions of the particles at the end of hybrid MAMBO version 1 at $I_{b}=150 \mu \mathrm{A}$ (Fig. 7a) and $I_{b}=$ $1 \mathrm{~mA}$ (Fig. 7b). Bunch length, energy spread and emittances are listed in Table V.
ICM [31]. (ii) In hybrid 2 a two-seater cryostat such as the ELBE cryostat used for the MESA main linac or the TRIUMF ARIEL e-Linac accelerator cryomodule ACM [31] could be used.

To reach a good comparability, the settings of MELBA and MAMBO 1 are identical to the normal conducting MAMBO. For all drift spaces inside the cryostat those of an ELBE module were assumed. Since the TESLA cavities are $\beta=1$ the slope of the phase slippage is always positive. The beam phases of the hybrids are plotted in Fig. 6. There it can be seen that the first and the last cell exhibits a different phase slip than the center cells. This is because those cells have a different length to allow coupling of the higher order modes (HOM) to the HOM dampers situated in the beam pipes [4].

Injection phase into the SRF cavity of hybrid 1 was chosen such that $\left|\phi_{\text {in }}\right| \approx\left|\phi_{\text {out }}\right|$ is achieved for near on-crest acceleration. The effective accelerating field was set to $E_{\text {acc }} \approx 3.8 \mathrm{MV} / \mathrm{m}$ which results in a final energy of approximately $5 \mathrm{MeV}$. The particle distribution is shown in Fig. 7. The corresponding bunch data is listed in Table V. The bunch exits the linac longitudinally converging. The amount of effective focusing can be adjusted by changing $\phi_{\text {in }}$ of the SRF cavity.

For hybrid 2, one can think of several modes of operation of the two SRF cavities. The first one is using both on-crest (hybrid 2a). Acceleration is split equally between both

TABLE V. Comparison of longitudinal bunch data of the hybrid MAMBO versions under investigation as found $0.1 \mathrm{~m}$ behind the end of the last rf section. Further the transverse rms emittances are given to judge the phase space evolution.

\begin{tabular}{|c|c|c|c|c|}
\hline \multirow[b]{2}{*}{$I_{b} \mathrm{~mA}$} & \multicolumn{2}{|c|}{ Hybrid 1} & \multicolumn{2}{|c|}{ Hybrid 2a } \\
\hline & 0.15 & 1 & 0.15 & 1 \\
\hline$\langle T\rangle \mathrm{MeV}$ & 5.09 & 5.09 & 5.05 & 5.05 \\
\hline$\Delta T_{\mathrm{rms}} \mathrm{keV}$ & 2.1 & 2.3 & 2.2 & 2.3 \\
\hline$\Delta T_{\mathrm{rms}} /\langle T\rangle 10^{-4}$ & 4.2 & 4.5 & 4.4 & 4.5 \\
\hline$\Delta \phi_{\mathrm{rms}}$ & 0.93 & 1.09 & 0.86 & 1.02 \\
\hline$\epsilon_{\mathrm{rms}}{ }^{\circ} \mathrm{keV}$ & 0.31 & 1.3 & 0.39 & 1.31 \\
\hline$\epsilon_{99 \%}{ }^{\circ} \mathrm{keV}$ & 4.27 & 20.79 & 5.19 & 20.67 \\
\hline$\epsilon_{100 \%}{ }^{\circ} \mathrm{keV}$ & 100.9 & 107.3 & 104 & 100.3 \\
\hline$\epsilon_{x, \mathrm{rms}} \mathrm{mm} \mathrm{mrad}$ & 0.2 & 1.34 & 0.2 & 1.34 \\
\hline \multirow[t]{2}{*}{$\epsilon_{y, \mathrm{rms}} \mathrm{mm} \mathrm{mrad}$} & 0.14 & 1.23 & 0.14 & 1.23 \\
\hline & \multicolumn{2}{|c|}{ Hybrid $2 b$} & \multicolumn{2}{|c|}{ Hybrid 2c } \\
\hline$\langle T\rangle \mathrm{MeV}$ & 5.08 & 5.08 & 5.1 & 5.1 \\
\hline$\Delta T_{\mathrm{rms}} \mathrm{keV}$ & 14.9 & 16.9 & 9.2 & 10.1 \\
\hline$\Delta T_{\mathrm{rms}} /\langle T\rangle 10^{-4}$ & 29.3 & 33.3 & 17.9 & 19.9 \\
\hline$\Delta \phi_{\mathrm{rms}}$ & 0.89 & 1.05 & 0.47 & 0.58 \\
\hline$\epsilon_{\mathrm{rms}}{ }^{\circ} \mathrm{keV}$ & 0.31 & 1.29 & 0.71 & 1.52 \\
\hline$\epsilon_{99 \%}{ }^{\circ} \mathrm{keV}$ & 4.24 & 21.27 & 10.35 & 22.37 \\
\hline$\epsilon_{100 \%}{ }^{\circ} \mathrm{keV}$ & 100.1 & 112.5 & 151 & 86.3 \\
\hline$\epsilon_{x, \mathrm{rms}} \mathrm{mm} \mathrm{mrad}$ & 0.2 & 1.34 & 0.2 & 1.34 \\
\hline$\epsilon_{y, \mathrm{rms}} \mathrm{mm} \mathrm{mrad}$ & 0.14 & 1.23 & 0.14 & 1.23 \\
\hline
\end{tabular}




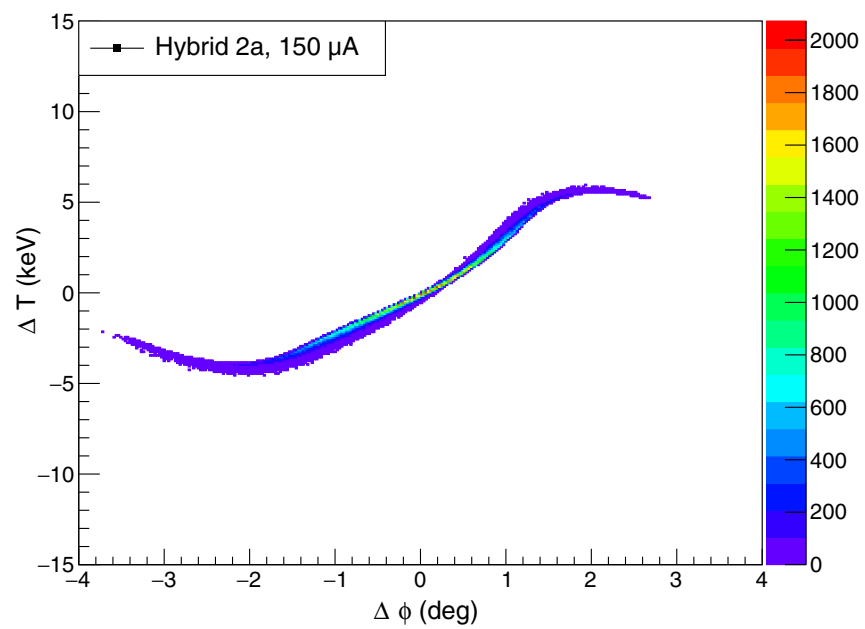

(a)

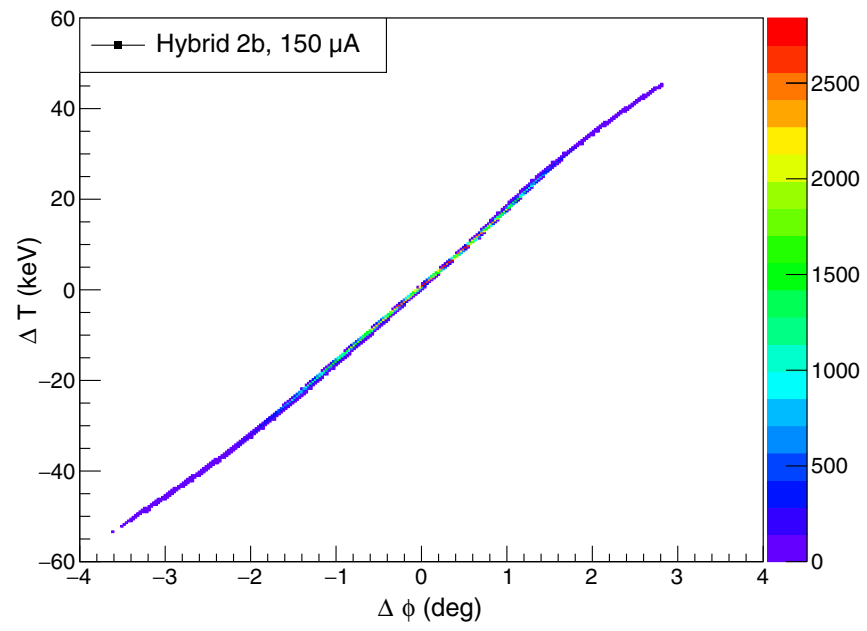

(c)

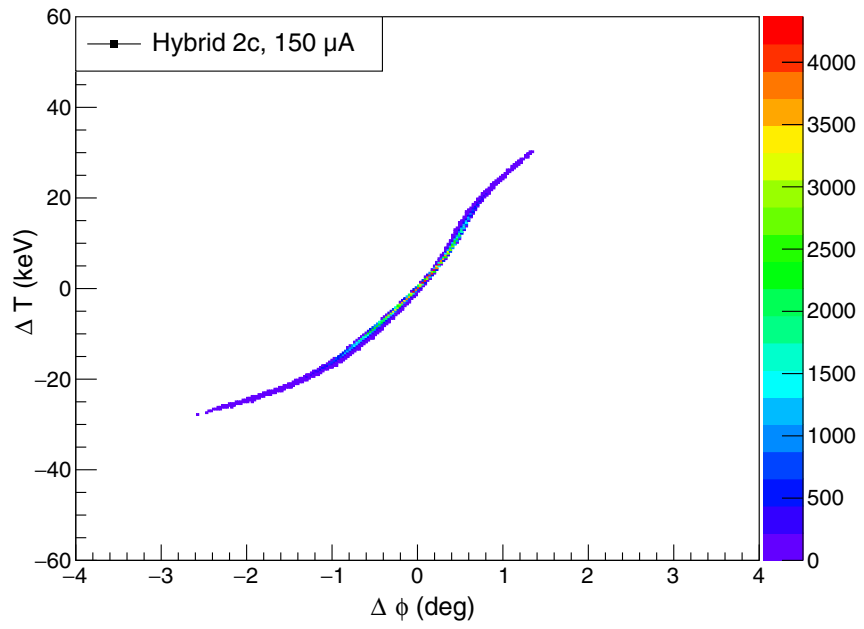

(e)

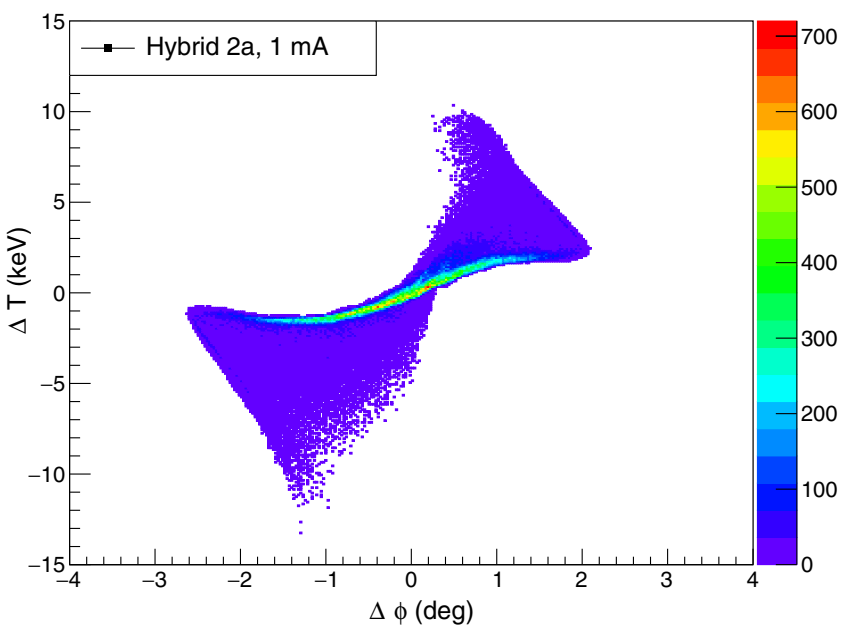

(b)

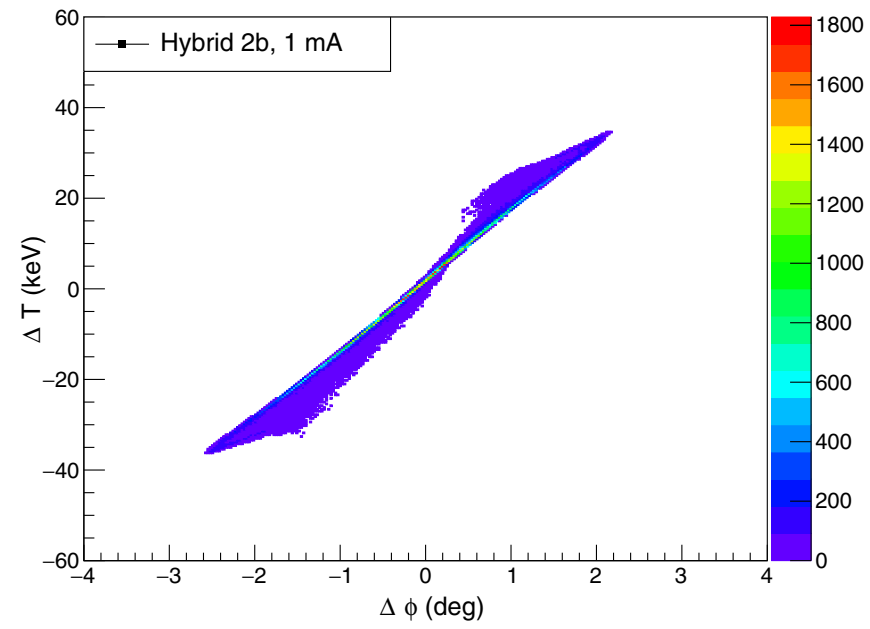

(d)

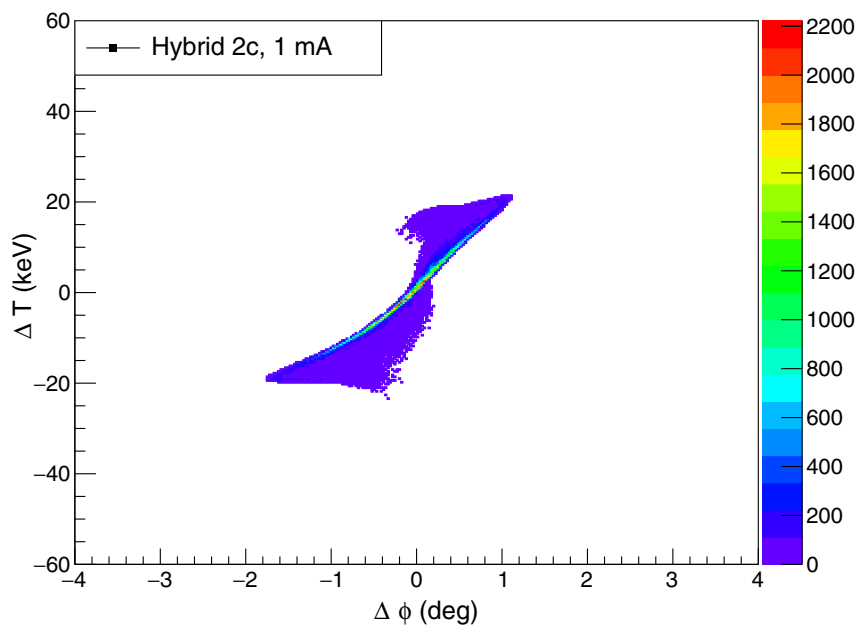

(f)

FIG. 8. Phase space distributions of the particles at the end of hybrid MAMBO version 2 at $I_{b}=150 \mu \mathrm{A}($ Figs. $8 \mathrm{a}, 8 \mathrm{c}$ and $8 \mathrm{e})$ and $I_{b}=1 \mathrm{~mA}$ (Figs. 8b, 8d and 8f). The bunch data of each operational mode is listed in Table V.

resonators running at a field gradient of $E_{\text {acc }} \approx 1.8 \mathrm{MV} / \mathrm{m}$. The other mode is to use one cavity for acceleration and the other one for additional focusing to have a shorter bunch to be transferred to MESA. If the first cavity is used for acceleration (hybrid 2b), the second one is set near zero crossing. This corresponds to a postbunching cavity added 
to hybrid 1 . While using the second resonator for acceleration (hybrid 2c), then the SRF cavity one cannot be run at zero crossing, because this results in a focal point inside the second cavity so the bunch exits the linac longitudinally diverging. So a less focusing phase needs to be chosen (see Fig. 6). In both cases, the accelerating cavity should operate around $E_{\text {acc }} \approx 3.8 \mathrm{MV} / \mathrm{m}$ and the focusing one at $E_{\text {acc }} \approx 0.8 \mathrm{MV} / \mathrm{m}$. The particle distributions resulting from the three modes of operation mentioned are shown in Fig. 8. The bunch data is also listed in Table V.

Comparing the emittances of the hybrid MAMBO with those at the end of MELBA (Table I) and with the emittances delivered by the normal conducting MAMBO it shows that in some settings the hybrid can almost preserve the emittances delivered by MELBA while in the normal conducting linac emittances grow by roughly $20 \%$ to $40 \%$. This can be explained by the higher field in the SRF cavities and thus higher longitudinal focusing on the one hand and on the other hand the shorter length of the hybrid so space charge forces diluting the emittance have less time to act on the bunch.

The phase space projections of hybrid 1 [Figs. 7(a) and 7(b)] and hybrid 2a [Figs. 8(a) and 8(b)] look quite similar. Also the corresponding bunch data in Table $\mathrm{V}$ are quite near to each other at $1 \mathrm{~mA}$ with hybrid 1 having about $20 \%$ smaller emittances at $150 \mu \mathrm{A}$. The additional focusing of the second SRF cavity of hybrid $2 \mathrm{~b}$ compared to hybrid 1 results in a strongly correlated and therefore less distorted phase space. It would be expected that the emittances of hybrid $2 \mathrm{~b}$ are smaller, but they are almost identical. To check whether there is less distortion of the phase space, one can put a very long drift after the last cavity and compare rms-bunch lengths at the focal points. Simulation for $I_{b}=150 \mu \mathrm{A}$ shows the bunch length of hybrid $2 \mathrm{~b}$ being about a factor of 6.6 smaller than that of hybrid 1 . A significantly shorter bunch at the focus is a clear sign of a less distorted phase space. The straightening of the phase space removes the accumulation of particles at the upper and lower ends of the energy spectrum without lengthening the bunch too much. It is most effective for the low current setting. As mentioned before, the energy spread of the injected beam is not as important as short bunches for the beam quality provided by the main accelerator. The energy spread of hybrid $2 b$ is increased by roughly a factor of 7 if compared to hybrid 1 or $2 \mathrm{a}$. This can be accepted in exchange for an improved energy distribution. The smoother energy spectrum generated with this operational mode could be very interesting for precision experiments. Hybrid 2c is not as effective in straightening the particle distribution as hybrid $2 \mathrm{~b}$. Although it provides the shortest bunches of all four, it produces the largest emittances. The emittances even exceed those of the normal conducting MAMBO.

Concerning transverse emittances the hybrid preaccelerator is comparable to the normal conducting one.
Summing up, in on-crest operation there is some difference between the hybrid using one or two TESLA cavities. The single cavity variant gives better beam quality at low current and has the advantage of less financial and less spatial needs. But the two cavity hybrid is more flexible, since different modes of operation providing interesting features can be employed. This makes it a more desirable solution. The sum of static and rf losses of the modules at $2 \mathrm{~K}$ is assumed at a conservative $Q_{0}=5 \times 10^{9}$ to be 9 to $12 \mathrm{~W}$.

\section{POSTBUNCHER FOR NORMAL CONDUCTING MAMBO}

To achieve a linear longitudinal phase space as provided by the hybrid $2 \mathrm{~b}$ also with a normal conducting MAMBO a short rf section MAMBO 5 could be positioned behind MAMBO 4. This section should operate at zero crossing.

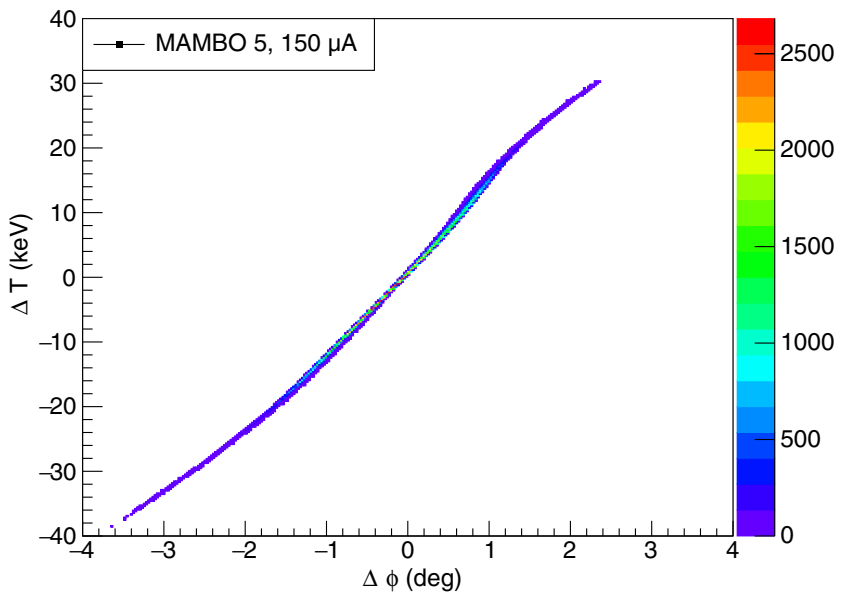

(a)

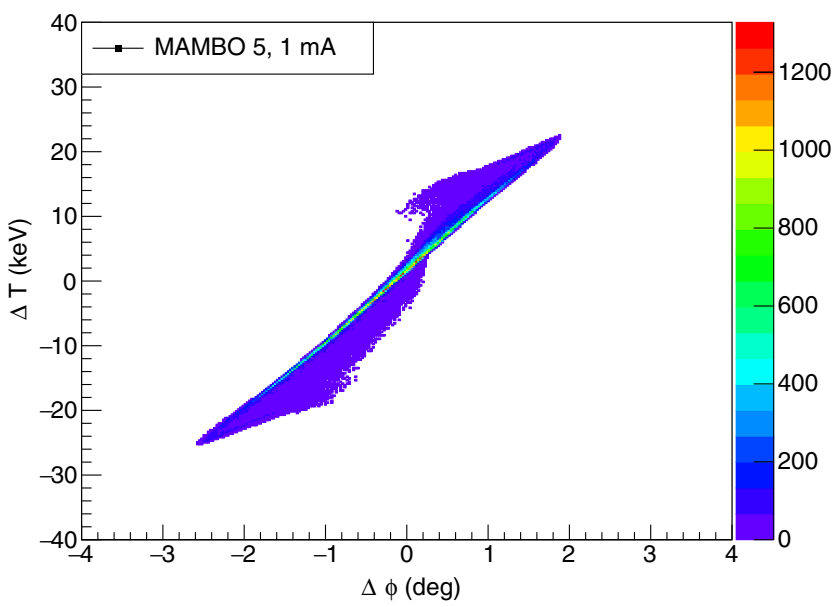

(b)

FIG. 9. Particle distributions in longitudinal phase space of different beam currents at the end of the normal conducting MAMBO version 2 with a post-buncher (Fig. 9a: $I_{b}=150 \mu \mathrm{A}$ and Fig. 9b: $I_{b}=1 \mathrm{~mA}$ ). 
TABLE VI. Longitudinal bunch data of the normal conducting MAMBO version 2 with a postbunching rf section. Additionally the transverse rms-emittance values are listed.

\begin{tabular}{lcc}
\hline \hline$I_{b} \mathrm{~mA}$ & 0.15 & 1 \\
$\langle T\rangle \mathrm{MeV}$ & 5.04 & 5.03 \\
$\Delta T_{\mathrm{rms}} \mathrm{keV}$ & 10 & 10.7 \\
$\Delta T_{\mathrm{rms}} /\langle T\rangle 10^{-4}$ & 19.8 & 21.3 \\
$\Delta \phi_{\mathrm{rms}}^{\circ}$ & 0.76 & 0.94 \\
$\epsilon_{\mathrm{rms}}{ }^{\circ} \mathrm{keV}$ & 0.41 & 1.31 \\
$\epsilon_{99 \%}{ }^{\circ} \mathrm{keV}$ & 5.74 & 21.17 \\
$\epsilon_{100 \%}{ }^{\circ} \mathrm{keV}$ & 116.2 & 82.3 \\
$\epsilon_{x, \mathrm{rms}} \mathrm{mm} \mathrm{mrad}$ & 0.22 & 1.3 \\
$\epsilon_{y, \mathrm{rms}} \mathrm{mm} \mathrm{mrad}$ & 0.15 & 1.25 \\
\hline \hline
\end{tabular}

For this a prototype that had been built for rf testing of the normal conducting MAMBO structure [3] could be used. It consists of $7 \mathrm{AC}$ and $6 \mathrm{CC}$ at $\beta_{5}=1$. It is critically coupled to the waveguide in order to reach $E_{\text {acc }}=1 \mathrm{MV} / \mathrm{m}$ with the $15 \mathrm{~kW}$ MESA prototype rf amplifier [32] so any limitations of the design would have been found during high power testing.

Since near zero crossing there is no beam loading that needs to be compensated by the coupling factor critical coupling is fine.

This setup has been simulated for $E_{\text {acc }} \approx 0.66 \mathrm{MV} / \mathrm{m}$. The input phase was chosen $\phi_{\text {in }} \approx-92^{\circ}$, so the middle cell was at $-90^{\circ}$. The particle distributions can be seen in Fig. 9 and the bunch data is listed in Table VI.

As expected the particle distribution looks less bent in the diagrams of Fig. 9 compared with Figs. 5(c) and 5(d). This is achieved on cost of the energy spread which is acceptable, as mentioned above. Bunch lengths again are not affected, also beam energy did not change considerably and emittances stayed the same within some percent. The effect of the postbuncher is again tested with a very long drift after MAMBO. One finds that the bunch length at the respective focal point is about a factor of 3.3 smaller for the beam at $I_{b}=150 \mu \mathrm{A}$ with postbuncher than it is without. The energy spread is increased by approximately the same amount. This confirms a decrease of the phase space distortion. So a postbuncher seems to be a good way to also achieve a more linear phase space distribution with the normal conducting linac.

\section{SUMMARY}

Two concepts of preaccelerators for MESA have been researched in different configurations. A fully normal conducting linac and a hybrid that employs normal conducting and off the shelf superconducting technology. It could be shown that low energy particles can be energy matched to standard SRF cavities by using a normal conducting graded- $\beta$ structure without a loss of beam quality. This removes the need for small projects to use either a high voltage gun exceeding several hundreds of kilovolts de voltage, or a SRF gun or to develop special SRF cavities for acceleration at low energy. As those approaches are technologically quite challenging, a basic normal conducting tank can be an easier solution.

Both concepts have been found suitable for MESA. Because of constraints in both the financial and the cryobudget the normal conducting option 2 has been chosen.

\section{REMARK}

The MESA upgrade scenario to $I_{b}=10 \mathrm{~mA}$ has not been researched, because this will most probably cause an upgrade of the energy of the particle source and therefore a redesign of the preaccelerator will be necessary. For this higher bunch charge special attention to beam breakup and HOM damping needs to be paid.

\section{ACKNOWLEDGMENTS}

The author would like to thank the colleagues S. Friederich, P. Heil and C. Matejcek for providing their work on STEAM and MELBA as a basis for the design of the preaccelerator concepts presented here.

[1] D. Becker, R. Bucoveanu, C. Grzesik et al., The P2 experiment, Eur. Phys. J. A 54, 208 (2018).

[2] S. Baunack, Low energy accelerators for high precision measurements, in Electromagnetic Interactions with Nucleons and Nuclei Conference (EINN2017), Paphos, Cyprus (2017), http://einnconference.org/2017/presentations/01_Nov/ W2/Baunack.pdf.

[3] F. Hug, K. Aulenbacher, R. Heine et al., Status of the MESA ERL-Project, in Proceedings of the 63th ICFA Advanced Beam Dynamics Workshop on Energy Recovery Linacs (ERL'19), Berlin, Germany (2019), pp. 14-17, https://doi.org/10.18429/JACoW-ERL2019MOCOXBS05.

[4] B. Aune, D. Bandelmann, D. Bloess et al., Superconducting TESLA cavities, Phys. Rev. ST Accel. Beams 3, 092001 (2000).

[5] J. Teichert, A. Büchner, H. Büttig, F. Gabriel, P. Michel, K. Möller, U. Lehnert, C. Schneider, J. Stephan, A. Winter, Rf status of superconducting module development suitable for cw operation: ELBE cryostats, Nucl. Instrum. Methods Phys. Res., Sect. A 557, 239 (2006).

[6] T. Stengler, K. Aulenbacher, R. Heine et al., Modified ELBE type cryomodules for the Mainz energy-recovering superconducting accelerator MESA, in Proceedings of the 17th International Conference in rf Superconductivity (SRF2015), Whistler, BC, Canada (2015), pp. 1413-1416.

[7] S. Friederich and K. Aulenbacher, Test electron source for increased brightness emission by near band gap photoemission, in Proceedings of the 6th International Particle Accelerator Conference (IPAC'15), Richmond, VA, USA (2015), pp. 1512-1514, https://doi.org/10.18429/JACoWIPAC2015-TUPWA044. 
[8] S. Friederich, K. Aulenbacher, and C. Matejcek, Vacuum lifetime and surface charge limit investigations concerning high intensity spin-polarized photoinjectors, J. Phys. Conf. Ser. 1350, 012045 (2019).

[9] S. Friederich, Entwicklung einer hochbrillanten Photoemissionsquelle für spinpolarisierte Strahlen, Ph.D. thesis, Institut für Kernphysik, Johannes Gutenberg-Universität, 2019, https://doi.org/10.25358/openscience-2370.

[10] C. Matejcek, K. Aulenbacher, S. Friederich, and L. Hein, Low energy beam transport system for MESA, in Proceedings of the 59th ICFA Advanced Beam Dynamics Workshop on Energy Recovery Linacs (ERL2017), Geneva, Switzerland (2017), pp. 20-25, https://doi.org/10.18429/ JACoW-ERL2017-MOPSPP008.

[11] C. Matejcek, K. Aulenbacher, and S. Friederich, Lowenergy beam transport system for MESA, J. Phys. Conf. Ser. 1350, 012027 (2019).

[12] V. Tioukine and K. Aulenbacher, Operation of the MAMI accelerator with a Wien filter based spin rotation system, Nucl. Instrum. Methods Phys. Res., Sect. A 568, 537 (2006).

[13] H. Braun, H. Herminghaus, and A. Streun, The gun/ chopper system for the Mainz microtron, in Proceedings of the 1st European Particle Accelerator Conference (EPAC'88), Rome, Italy (1988), pp. 997-999.

[14] V. Shvedunov, H. Euteneuer et al., Design of a prebuncher for increased longitudinal capture efficiency of MAMI, in Proceedings of the 5th European Particle Accelerator Conference (EPAC'96), Sitges, Spain (1996), pp. 1502-1504.

[15] I. Alexander, K. Aulenbacher, V. Bechtold, B. Ledroit, and C. Matejcek, Diagnostic test-beam-line for the MESA injector, in Proceedings of the 56th ICFA Advanced Beam Dynamics Workshop on Energy Recovery Linacs (ERL'15), Stony Brook, NY (2015), pp. 91-96.

[16] F. Schlander, K. Aulenbacher, R. Heine, and D. Simon, Investigation of cryomodules for the Mainz energyrecovering superconducting accelerator MESA, in Proceedings of the 5th International Particle Accelerator Conference (IPAC 2014), Dresden, Germany (2014), pp. 2505-2507, https://doi.org/10.18429/JACoW-IPAC2014WEPRI013.

[17] K. Aulenbacher, C. Nachtigall et al., The MAMI source of polarized electrons, Nucl. Instrum. Methods Phys. Res., Sect. A 391, 498 (1997).

[18] K. Aulenbacher, Polarized beams for electron accelerators, Eur. Phys. J. Special Topics 198, 361 (2011).

[19] M. Tanabashi et al. (Particle Data Group), Review of particle physics, Phys. Rev. D 98, 030001 (2018).

[20] M. Dehn, K. Aulenbacher, R. Heine, H. J. Kreidel, U. Ludwig-Mertin, and A. Jankowiak, The MAMI C accelerator. The beauty of normal conducting multiturn recirculators, Eur. Phys. J. Special Topics 198, 19 (2011).

[21] R. Heine and K. Aulenbacher, Injector LINAC for the MESA facility, in Proceedings of the 4th International Particle Accelerator Conference, IPAC'13, Shanghai, China, 2013 (JACoW, Shanghai, China, 2013), pp. 2150-2152.

[22] R. Heine, K. Aulenbacher, S. Friederich, C. Matejcek, and F. Schlander, Further investigations on the MESA injector, in Proceedings of the 6th International Particle Accelerator Conference (IPAC'15), Richmond, VA (2015), pp. 1515-1517.

[23] R. Heine, K. Aulenbacher, L. Hein, and C. Matejcek, Current status of the milliampere booster for the Mainz energy-recovering superconducting accelerator, in Proceedings of the 7th International Particle Accelerator Conference (IPAC'16), Busan, Korea (2016), pp. 1741-1743.

[24] J. H. Billen, PARMELA (2005).

[25] J. H. Billen and L. M. Young, POISSON/SUPERFISH on PC compatibles, in Proceedings of the 15th Particle Accelerator Conference, PAC'93, Washington, DC, 1993 (IEEE, New York, 1993), pp. 790-792.

[26] I. Antcheva, M. Ballintijn, B. Bellenot et al., ROOT A C++ framework for petabyte data storage, statistical analysis and visualization, Comput. Phys. Commun. 180, 2499 (2009).

[27] D. E. Nagle, E. A. Knapp, and B. C. Knapp, Coupled resonator model for standing wave accelerator tanks, Rev. Sci. Instrum. 38, 1583 (1967).

[28] E. A. Knapp, B. C. Knapp, and J. M. Potter, Standing wave high energy linear accelerator structures, Rev. Sci. Instrum. 39, 979 (1968).

[29] H. Euteneuer, H. Braun, H. Herminghaus, R. Klein, H. Schöler, and T. Weis, The injector LINAC for the Mainz microtron, in Proceedings of the 1st European Particle Accelerator Conference (EPAC'88), Rome, Italy (1988), pp. 550-552.

[30] F. Hug and R. Heine, Injector linac stability requirements for high precision experiments at MESA, J. Phys. Conf. Ser. 874, 012012 (2017).

[31] N. Muller, R. E. Laxdal, P. Harmer et al., TRIUMF's injector and accelerator cryomodules, in Proceedings of the 17th International Conference on rf Superconductivity (SRF2015), Whistler, BC, Canada (2015), pp. 1409-1412.

[32] R. Heine and F. Fichtner, The MESA $15 \mathrm{~kW} \mathrm{cw} 1.3 \mathrm{GHz}$ solid state power amplifier prototype, in Proceedings of the 9th International Particle Accelerator Conference (IPAC'18), Vancouver, Canada (2018), pp. 4216-4218, https://doi.org/10.18429/JACoW-IPAC2018-THPMF063. 